\title{
Study on Software project management system based on DFD Editor version
}

\author{
Jinhai Zhang \\ Marine college of Shandong jiaotong university, Weihai, Shandong,China
}

Keywords: CSCW, data modeling, time stamp, multicast, parameter optimization

\begin{abstract}
Software process management system, the needs analysis is the basis for all work and source, is a top priority. Requirements analysis, software developers, project leaders and team members and between the software developer and software on the client side, requires adequate and convenient communication. Proposes a collaboration to resolve data synchronization over multiple versions system conflict solution for solving the DFD model during synchronous collaboration has developed a comprehensive programme. CSCW data flow modeling based on multiple versions is innovation.
\end{abstract}

\section{Introduction}

Software engineering and project management techniques have been developed for several years, is mature and profound subjects. In the 1960 of the 20th century "software crisis" since the emergence of people has been seeking more advanced software technology to solve new problems in the software field, the concept of software engineering first appeared in the 1968 NATO (North Atlantic Treaty Organization) meeting. The third era of software development, software industrialization era to CMM maturity model and increasingly accepted as a sign for the market, to the mid-90 software process maturity of technology and technology of object-oriented technologies, component based development, have gradually emerged. Real software for industrial production of this trend and the trend of the world, attracted great attention of software businesses and departments concerned.

Software development process is a group process, particularly for large and complex systems development and cooperation needed by many people to complete. According to statistics, in large-scale projects major systems developers need to spend $70 \%$ time work together with other persons, group activities to take up $85 \%$ in large-scale software systems development costs. These data show: System development of most of the consumption of resources come from members of the development team in coordination and communication. If you want to significantly improve the efficiency of software developers (which is obviously the common goal of all software development and producers), must be assisted in the CASE of software development tools that support collaborative work.

For software developers, and software developers and software on the client side provides a good medium of communication between the staff. First of all, the system allows multiple users to synchronize Exchange online, what you see is what I see in the mode, the communication more intuitive full, real-time, secondly, multi-versioning method makes use of the system to accommodate the views of all participants - even if that advice is conflicting. Such a system makes collaboration more full and friendly, software developers and customers can communicate online in real time, not because of conflicting intentions and busywork or some of the people do not have access to the reservation. This system fills an existing CASE tool no good AC and does not implement good conflict resolution vacancies.

\section{CASE and CSCW technology}

Software process improvement is a core engineering software management issues. More than 50 years to calculate the development of awareness to efficient, high quality and low cost development of software, software production processes must be improved. Software management engineering 
through has a article from 70 generation began to structure of analysis and design, and structure of review, and structure of programming and structure of test to 90 generation medium-term to process mature model CMM, and individual software process PSP and group software process TSP for logo of to process for center toward software process technology of mature and oriented object technology, and component technology of development for based of real software industrialization production of road. Software production to improve software process-centric, worldwide software industry sooner or later has to go way. Software have been or are going through "the maturity of the software processes" and "industrialization of the software" a gradual transition. Standard software process is necessary for the industrialization of software.

CMM (Capability Maturity Model capability maturity model) software management is the essence of a part. It is for software organizations in the definition, implementation, measuring, controlling and improving its software processes at various stages in the process of describing, evolving through 5 levels to evaluate the software production history and the present situation.

To guarantee software development organization in the implementation CMM assessment of effectiveness, on must in carried out CMM assessment of while, research, and established and perfect CMM in the by to out of software needs management, and software project planning, and software track and supervision, and software (child) contract management, and software process document management, and software standardization, and software quality guarantee, and software configuration management, and software maintenance, and integrated software management, and software products engineering of, and organization coordination, and peer experts review, and prevention defects, and technology change management, and process change management, and Organizational process management process management and training of 18 key process areas, such as management practices.

Traditional CASE tools generally work in a stand-alone environment, information integration. Due to the lack of communication on the management of collaborative mechanisms, it is difficult to realize the requirement of full and effective communication during the design process. With the rapid development of network technology, CSCW technology began in the late 80 experts and scholars of note and become a specialized field of study, CSCW technology into the CASE tool has become a hot topic of research in the field of software engineering.

In CSCW system in the, more user collaboration main involved two a problem: a is established including external environment and collaboration members of collaboration scene, for collaboration members provides and external environment and other collaboration members Zhijian of information exchange, this is can antibody for groups perception problem; II is determine members Zhijian collaboration behavior of control mechanism, solution collaboration system in the collaboration relationship of produced and description, and the members on collaboration of joined and exit and collaboration behavior of conflict elimination, problem, can antibody for collaboration control problem.

\section{Data flow diagram drawing model based on multi-versioning method}

When multiple users on the same property for the same object (in the DFD editing system was the same entity object with a property such as position, within the text, and so on) produce different actions when we have said they intend to produce a conflict. Solution to the conflict there are several:

(1) empty action programme: in this scenario, once clashed prior to operation of the operating system back to the conflict, namely that these conflicting operation has not happened. Such clashes as long as operations, any user action intentions cannot be saved.

(2) retained any of the operational programme: clashes after the operation, retain any results arising from the operation of the system, so that once the conflict, there is only one operation results can be saved.

(3) version scenario: all of the conflicting operation after the conflicting operation produces a result preserved, generated for each different actions result in a new version. Version proposed by 
SUN, this method can be made to resolve the conflict in the system when there is a conflict in operation, each conflict is saved down action has the same effect.

Contrast these conflict resolution, multi-versioning scheme while preserving the designers intended advantages are clear. Multi-versioning scheme system can guarantee that in the event of conflict after all the user's intentions are fully preserved. In this way, each user in the event of conflicts when other users want to do be able to do so knowing, so help them take appropriate contingency measures.

In many versions and many copies of graphic editing system based on object, object identification scheme must have three characteristics:

- uniqueness: every object in a site must have a unique identifier.

- traceability: copied from object g g can use identifiers to track multiple versions of objects.

- consistency: copies of the same object in different sites must have the same identity.

First ensure the different objects in the same site can distinguish each other. Tracking features ensure that multiple versions of the same object can be identified in this version of the find. Consistency guarantees that multiple copies of the same object with the same ID, so that a copy of all of the changes can be applied to the replica.

\section{The versions of software project management system design and development}

Software development of integrated project management including: monitoring, requirements management, project planning, project management, configuration management, contract management and 7 much of the daily work module. Passage of the 7 modules on a variety of software development to provide full monitoring and support. Collaborative drawing in demand management module provides functions to support fully synchronized online graphical communication and consultation, is a feature of the system. This DFD drawing together with DFD editing system is different in the past, the main purpose and function is to allow participants in the data flow diagram design discussions, allowing multiple conflicting views coexist, and also discuss the intention to enable designers to show more fully. Used. Net Enterprise template for development makes the entire project structure is clear, strong and reusable, fully learned today to promote the idea of software engineering projects, making the entire development in order for the future development of a similar program established a reusable structure.

In the requirements of the process, because the domain of knowledge differences between the customer and technician, everyone expressed understanding differences in the field of design differences between design intent and negotiation problems arise. A good graphical interface to synchronous collaboration tools are excellent tools for friendly consultations. At the time of this function, the difficulty: (1) good reflection, (2) stable system performance integrity, (3) good conflict resolution approach is the difficulty of synchronous collaboration tool. Among them, in order to achieve good response speed as well as the development of a more stable system, we take the multicast delivery information as well as maintain a copy of the database for each site, rejected the traditional patterns of $\mathrm{B} / \mathrm{S}$ and leads to the following difficulties: (1) to ensure the centralized operation, (2) operation causes confusion, (3) operations intended to keep. Therefore, solving these challenges become the focus of the system.

In traditional development models, software analysis phase of the software development plays a very important role. Structured analysis method using a "top-down decomposition layer by layer" approach, reasonably complex system break down layer by layer, until each subsystem can be a simple, clear expression and understanding so far. SA obtained the system specification is made up of a set of hierarchical data flow diagram of a data dictionary. Effective software analysis methods must enable users and designers can communicate fully, requires everyone, including users and designers can understand, and can be refined and improved comments intermediary, making software simple, intuitive and complete. SA method and structural design of nature by the SD interface, from requirements analysis, systems analyst can smoothly transition to preliminary design stage. 
The logical architecture of systems using data-centric, namely: public interface between data formats as tools, tools can be simply comply with the format of this public interaction. Point-to-point communication, each host is both a client and a server. We can fit the following several aspects:

- suitable for the establishment of an open system environment. User can arbitrarily create a team collaboration environment by distributed environment, restrictions on the user's physical location and the number of requests is very small (of course, the speed will slow down as the surge).

- each machine has application, access to the local copy of the database without $\mathrm{C} / \mathrm{S}$ or $\mathrm{b} / \mathrm{s}$ structure appears in the server is overloaded.

- appropriate to establish a relatively stable environment, does not appear because the server node crashes, the entire system may not function properly. No matter what node there is a problem, other machines can function properly, will not affect the work. (If problems with node system administrator, system dynamic permissions give administrators permissions given can be used most of the time users. )

This model requires that all relevant actions executed in the order of their natural occurrence, but for any operation that is not associated with no such restrictions. In order to achieve a good response, this model allows any local operations performed locally at once. This model has been successfully used in text editing systems. In order to achieve the causality preservation, well-known logical time stamp is used to capture causality between actions, and selectively delaying some confusing causal order to reach operation. For high response limitations supported intention save and centralized, a good consistency control called operational transformation was adopted. Novelty this operational transformation mechanism is that it allows independent operations in any order, but it guarantees the operation of eventual consistency and intent of these actions to save. But there are indications that some action result method intended to save the conversion is not complete by any conventional serial protocols. Our findings based on logical causality preservation technology of the clock is a general technique, not only can you use to text editing, you can also use graphics editor.

\section{References}

[1] R Broudes, S Zabele, Requirement for Multicast Protocol, RFC 1458, 2003.5

[2] Campbell, J.D.Consistency maintenance for real-time collaborative diagram development. Ph.D.thesis, Department of Information Science, University of Pittsburgh, Pittsburgh. PA. USA. 2002.

[3] Liyin Xue, Mehmet A.Orgun. Locking without requesting a lock: Aconsistency maintenance mechanism in Internet-based real-time group editors.Journal of Parallel and Distributed Computing, 2005,1-14.

[4] Jennings N.R. and Wooldridge J.M. Applications of Intelligent Agents. In: Proceedings of Agent Technology, edited by Jennings N.R.and Wooldridge J.M. Springer Press,1998,3-28

[5] Gaertner J.Participatory Design in Consulting.Computer Surpported Coop erative Work, 2008, $7(3 / 4): 273-289$

[6] Kensing F.and Blomberg J.Participatory Design:Issues and Concern, Computer Surpported Cooperative Work, 1998,7(3/4):167-185

[7] Mambrey P. Mark G.and Pankoke-Babatz U.User Advocacy in Participatory Design: Designers Experience with a New Communication Channel. Computer Surpported Cooperative Work, 2008, 7(3/4): 291-313 